\title{
Tyrosine Phosphorylation of Focal Adhesion Kinase and Paxillin Regulates the Signaling Mechanism of the Rapid Nongenomic Action of Dexamethasone on Actin Cytoskeleton
}

\author{
Sevasti B. Koukouritaki, ${ }^{1}$ Achille Gravanis, ${ }^{2}$ and \\ Christos Stournaras ${ }^{1}$ \\ Departments of ${ }^{1}$ Biochemistry and ${ }^{2}$ Pharmacology, School of \\ Medicine, University of Crete, Heraklion, Greece \\ Accepted September 17, 1999.
}

\begin{abstract}
We have previously shown that dexamethasone (DEX) stimulates rapid polymerization of actin and stabilization of microfilaments in human endometrial adenocarcinoma cells. As the content of total cellular actin and the concentration of the actin transcript did not change, we concluded that polymerization of actin by glucocorticoids involves nongenomic mechanisms. However, the signaling events by which the latter is achieved remain unknown. In the present study we evaluated whether tyrosine phosphorylation is required for the rapid, nongenomic DEX effect on actin assembly. In cells preincubated with the tyrosine kinase inhibitors, genistein or erbstatin analogue (EA), before adding DEX the G-/total actin ratio remained unchanged, whereas DEX in the absence of both inhibitors reduced the ratio by $25 \%$. In addition, when cells were preincubated with the protein tyrosine phosphatase inhibitor pervanadate and subsequently incubated with DEX, the G-/total actin ratio was dramatically reduced by $65 \%$. Furthermore, DEX increased transiently the levels of tyrosine phosphorylation of focal adhesion kinase (FAK) and paxillin within 2 to $15 \mathrm{~min}$, without a change in their expression levels. Pervanadate mimicked this effect of DEX and enhanced
\end{abstract}

tyrosine phosphorylation of both proteins. In addition, when cells were exposed to the anticytoskeletal agent cytochalasin B, the basal levels of tyrosine phosphorylation of both proteins were reduced. This effect was reversed by DEX, indicating that actin cytoskeleton integrity is required for the effect of DEX on tyrosine phosphorylation of FAK and paxillin. Finally, we documented enhanced expression of the Ras-related GTPbinding protein Rho-B after long-term (12- and 24-hr) treatment with DEX, whereas Rho-B levels remained unchanged after short-term (3- and 6-hr) treatment. Our observations demonstrate a novel mechanism through which the rapid nongenomic effect of DEX on actin assembly requires tyrosine phosphorylation of the cytoskeleton-associated proteins FAK and paxillin. We also propose that the DEX-induced actin polymerization may constitute a mechanism for transduction of signals resulting in tyrosine phosphorylation of FAK and paxillin. Moreover, the enhanced Rho-B levels observed after long-term treatment with DEX imply a mechanism for the well-described, long-term effects of glucocorticoids on actin cytoskeleton.

\section{Introduction}

Steroid hormones may affect cell function not only through genomic events, but also through nongenomic pathways of rapid onset (1). The mechanisms by which these nongenomic effects are mediated have not yet been clarified. The actin microfilament system is a likely mechanism

Address correspondence and reprint requests to: Dr. Christos Stournaras, Department of Biochemistry, School of Medicine, University of Crete, GR-71110 Heraklion, Greece. Tel: 3081 394563; Fax: 3081 394530; E-mail: cstourn@med.uoc.gr
$(2,3)$. Several studies have shown that rapid changes in actin polymerization may contribute to the way in which hormones induce rapid cellular events such as secretion, exocytosis, or volume regulation $(4,5)$.

We have recently reported that glucocorticoids exert rapid, nongenomic stimulatory effects on actin assembly and microfilament stabilization in Ishikawa human endometrial cells, involving cAMP-dependent regulatory pathways (2). In addition, we found that glucocorticoid induction of actin polymerization, as determined 
by the levels of rhodamine phalloidin-labeled F-actin in cells exposed to dexamethasone (DEX) and by confocal laser scanning microscopy, was not followed by changes in actin gene transcription (3). The molecular mechanism regulating the rapid, nongenomic glucocorticoid action on actin cytoskeleton remained unknown, although the observed changes in cAMP levels suggested quickly responding signaling events.

It is well known that tyrosine phosphorylation is essential for cytoskeleton microfilament assembly $(6,7)$. Tyrosine-phosphorylated proteins are located predominantly where actin filaments terminate at sites of cell-to-cell and cellto-substrate adhesion and are understood to regulate cellular adhesions and filamentous (F) actin organization in many cell types. Moreover, tyrosine phosphorylation of specific cytoskeletal proteins is a mechanism underlying the assembly of focal adhesions between cells and the extracellular matrix (ECM) (8). Focal adhesions are not only sites of structural linkage between the ECM and the cytoskeleton but also of transmembrane signaling $(9,10)$. Proteins that become phosphorylated on tyrosine in association with microfilament assembly and formation of focal adhesions include focal adhesion kinase (FAK) and paxillin (11). Phosphorylation of FAK or paxillin on tyrosine residues occurs in response to engagement of the integrin receptor with ECM proteins $(12,13)$, as well as in response to extracellular signals such as hormones (13-15), growth factors $(16,17)$, and regulatory peptides (18). FAK, in turn, phosphorylates paxillin $(19,20)$. Moreover, it is well established that signal transduction pathways leading from membrane receptor activation to actin reorganization involve small GTPases (21-23). It is well documented that Rho, rac, and cdc42 GTPases regulate the organization of the actin cytoskeleton (24), while Rho-B regulates the activity of FAK and paxillin, and stimulates formation of focal adhesions between cells and their substratum $(21,25)$.

In the present study, we explored putative mechanisms mediating the rapid effect of glucocorticoids on actin assembly. We explored whether tyrosine phosphorylation is required for DEX-induced actin assembly, using the tyrosine kinase inhibitors genistein and ebstatin analogue (EA), and the tyrosine phosphatase inhibitor pervanadate. We show here that actin assembly induced by DEX is associated with an elevation in tyrosine phosphorylation levels of FAK and paxillin. We also show that DEX restores tyrosine phosphorylation of FAK and paxillin following disruption of the cytoskeletal assembly by cytochalasin B (CB). Finally, we assessed changes in expression of the Ras-related GTP-binding protein Rho-B after short- and long-term incubations with DEX. As in our in vitro model, we used the Ishikawa human adenocarcinoma cell line, which expresses functional glucocorticoid receptors and proved to be a reliable tool in our previous reports $(2,3)$.

\section{Materials and Methods}

\section{Reagents}

Fetal bovine serum (FBS), minimum essential medium (MEM), L-glutamine, penicillin, and streptomycin were from Gibco (Life Technologies, Gaithersburg, MD). Dulbecco's modified Eagle medium (DMEM)/Ham's F-12, bovine serum albumin (BSA), genistein, sodium orthovanadate, protein G Sepharose, and cytochalasin B were from Sigma (St. Louis, MO). Erbstatin analogue (EA; methyl 2,5-dihydroxycinnamate) was from Biomol Research Labs (Plymouth Meeting, PA). Monoclonal antibodies against FAK and phosphotyrosine were purchased from Upstate Biotechnology (Lake Placid, NY), and monoclonal antibody against paxillin was from Transduction Laboratories (Santa Cruz, NM). The polyclonal antibody against Rho-B was from Santa Cruz Biotechnology (Santa Cruz, CA). The enhanced chemilluminescence (ECL) Western blotting kit, the horseradish peroxidase-conjugated sheep anti-mouse IgG, and the donkey anti-rabbit IgG were purchased from Amersham (Arlington Heights, IL). The Bio-Rad protein assay kit II (500-0002) was from BioRad Laboratories (Palto Alto, CA). All other chemicals were obtained from usual commercial sources at the purest grade available.

\section{Cell Culture}

Ishikawa cells were established as a permanent cell line from a well-differentiated endometrial adenocarcinoma (26). The cells were cultured in $25-$ or $75-\mathrm{cm}^{2}$ flasks in DMEM containing $15 \%$ FBS. Cultures were performed in a $5 \% \mathrm{CO}_{2}-$ $95 \%$ air atmosphere at $37^{\circ} \mathrm{C}$. At near confluence, the cells were washed twice and cultured for 12 hr in DMEM/Ham's F-12 in the absence of serum, but supplemented with $10 \mathrm{mM}$ L-glutamine, $15 \mathrm{mM}$ HEPES, and $1 \%$ antibiotic-antimycotic solution to final concentrations of 100 
$\mathrm{U} / \mathrm{ml}$ penicillin and $100 \mu \mathrm{g} / \mathrm{ml}$ streptomycin. For measurement of the cellular G- and total actin content and for immunoprecipitation and immunoblotting experiments, cells were washed twice with cold phosphate-buffered saline (PBS) and removed from flasks using scrapers.

\section{Use of DEX and Experimental Design}

DEX was dissolved in absolute ethanol and introduced in cultured Ishikawa cells, serum starved for $12 \mathrm{hr}$, at $10^{-7} \mathrm{M}$ for defined time periods $(2,15$, and $30 \mathrm{~min}$ and $1,3,6,12$, and 24 $\mathrm{hr}$ ). In control incubation experiments, the DEX vehicle (absolute ethanol) was introduced for identical time periods. To explore whether tyrosine phosphorylation is required for microfilament assembly induced by DEX, cells were pretreated with two different tyrosine kinase inhibitors: genistein $\left(4^{\prime}, 5,7\right.$-trihydroxyisoflavone), which competitively inhibits ATP binding to the kinase domain of several tyrosine kinases (27) at $100 \mu \mathrm{g} / \mathrm{ml}$ for $90 \mathrm{~min}$, and erbstatin analogue (methyl 2,5-dihydroxycinnamate), which structurally mimics the tyrosyl moiety and thus shows more specificity toward tyrosine kinases (28) at $1 \mu \mathrm{M}$ for $3 \mathrm{hr}$. Cells were also pretreated for $30 \mathrm{~min}$ with sodium orthovanadate (1 mM) in conjunction with hydrogen peroxide ( $3 \mathrm{mM})$, (pervanadate or vanadyl hydroperoxide, $\left[\mathrm{V}^{(4+)}\right.$ $\mathrm{OOH}$ ), - which acts as a potent inhibitor of a variety of cellular phosphatases in vivo (7). To explore whether an intact cytoskeleton is required for tyrosine phosphorylation of FAK and paxillin induced by DEX, cells were pretreated with $\mathrm{CB}$, which selectively disrupts the network of the actin filaments. In these experiments, $\mathrm{CB}$ was dissolved in absolute ethanol and introduced in cultured cells to a final concentration of 1.2 $\mu \mathrm{M}$ for $2 \mathrm{hr}$. In control incubation experiments, the $C B$ vehicle (absolute ethanol) was introduced for the same time period. At completion of incubations with DEX, cells were processed either for measurement of G- and total actin concentration or for assessment of expression and tyrosine phosphorylation of FAK and paxillin, or for assessment of Rho-B expression. These methods are detailed below.

\section{DNaseI Inhibition Assay for Determination of Intracellular Actin}

Monomeric (G-) and total actin concentrations were measured using the DNase I inhibition assay with minor modifications, as previously de- scribed $(5,29)$. The DNase I activity was monitored continuously at $260 \mathrm{~nm}$ in a Perkin-Elmer Lamda 15 double-beam spectrophotometer. The intracellular actin content was quantified by reference to a standard curve of the inhibition of DNase I activity, made using rabbit muscle Gactin, isolated as previously described (30). A linear relationship was observed in the range of $25-70 \%$ inhibition of DNase I activity. For measurement of total actin levels, aliquots of cell lysate were diluted two to three times with lysis buffer (see below) and then incubated for $20 \mathrm{~min}$ on ice with an equal volume of guanidine- $\mathrm{HCl}$ buffer $(1.5 \mathrm{M}$ guanidine $-\mathrm{HCl}, 1 \mathrm{M}$ sodium acetate, $1 \mathrm{mM}$ ATP, $20 \mathrm{mM}$ Tris- $\mathrm{HCl}, \mathrm{pH} \mathrm{7.5)}$ to depolymerize the F-actin to monomeric G-actin. G- and total actin content were expressed per total cell protein content as measured by a colorimetric assay (Bio-Rad Protein Assay).

\section{Immunoprecipitation}

Cells were lysed on ice with cold lysis buffer consisting of $50 \mathrm{mM}$ HEPES, pH 7.5; $150 \mathrm{mM}$ $\mathrm{NaCl} ; 1.5 \mathrm{mM} \mathrm{MgCl}_{2} ; 1 \mathrm{mM}$ EGTA; $10 \%$ glycerol; $1 \%$ Triton $\mathrm{X}-100 ; 1 \mu \mathrm{g} / \mathrm{ml}$ leupeptin; $1 \mathrm{mM}$ PMSF; and $200 \mu \mathrm{M}$ sodium orthovanadate. Cell material was scrapped off the flasks and allowed to lyse for an additional $30 \mathrm{~min}$ on an orbital shaker at $4^{\circ} \mathrm{C}$. Samples were subsequently centrifuged for $30 \mathrm{~min}$ at $14,000 \times g$ at $4^{\circ} \mathrm{C}$ and supernatants were transferred to fresh centrifuge tubes. Protein concentrations were determined using a colorimetric assay (Bio-Rad Protein Assay). Cell lysates containing equal amounts of protein were incubated either with anti-FAK monoclonal IgGl or with anti-phosphotyrosine monoclonal IgG2bk at $4^{\circ} \mathrm{C}$ overnight. Antigenantibody complexes were then precipitated by agitation for $2 \mathrm{hr}$ at $4^{\circ} \mathrm{C}$ with washed protein $\mathrm{G}$ Sepharose bead slurry. Beads were collected by pulse centrifugation $(14,000 \times g, 5 \mathrm{sec})$ and washed three times with lysis buffer. Immunoprecipitates were solubilized by dissolving in gel sample buffer and were boiled for $5 \mathrm{~min}$. Beads were collected by centrifugation and SDS-PAGE was performed with the supernatant fraction.

\section{Immunoblotting}

Proteins in cell lysates were resolved on acrylamide mini-gels (100 V, constant voltage) using a mini-Protean II Electrophoresis Cell, and electroblotted to nitrocellulose membranes. To resolve FAK-containing immunoprecipitates, the 


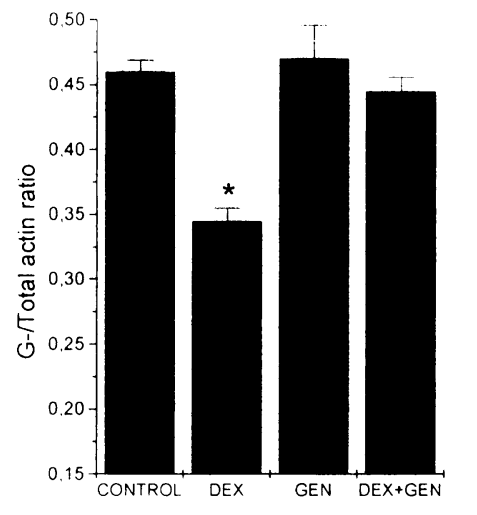

A

Fig. 1. Determination of G-/total actin ratios in serum-starved Ishikawa cells. (A) Cells were preincubated for $90 \mathrm{~min}$ with genistein (GEN, 100 $\mu \mathrm{g} / \mathrm{ml}$ ), followed by a 15 -min treatment with $10^{7}$ $M$ dexamethasone (GEN + DEX). (B) Cells were preincubated for 3 hr with erbstatin analogue (EA, I $\mu \mathrm{M})$, followed by a 15 -min treatment with $10^{-7} \mathrm{M}$ DEX (EA + DEX). (C) Cells were preincubated for

immunoprecipitates obtained as described above were run on 4-20\% gradient acrylamide gels, while a $12 \%$ acrylamide gel was used for resolving paxillin-containing immunoprecipitates and for immunoblotting Rho-B. Blots were blocked for $1 \mathrm{hr}$ at room temperature with $5 \%$ nonfat dry milk in T-TBS (Tris-buffered saline; $10 \mathrm{mM}$ Tris, pH 7.5; $100 \mathrm{mM} \mathrm{NaCl} ; 0.1 \%$ Tween-20) prior 10 incubation with anti-FAK or anti-paxillin monoclonal antibody. For immunoblotting of Rho-B, blocking was performed for $1 \mathrm{hr}$ at room temperature with $5 \%$ nonfat dry milk in T-TBS containing $0.05 \%$ Twen-20. Membranes were then probed for 1 hr at room temperature with one of the following primary antibodies: monoclonal anti-FAK antibody at $1 \mu \mathrm{g} / \mathrm{ml}$ diluted in T-TBS; monoclonal anti-paxillin antibody at 1:4000 dilution in blocking buffer; polyclonal anti-Rho-B antibody at $1 \mu \mathrm{g} / \mathrm{ml}$ diluted in blocking buffer. After incubation with the primary antibody, membranes were extensively washed with several changes of T-TBS $(0.1 \%$ Tween-20 for antiFAK and anti-paxillin blots, $0.05 \%$ Tween-20 for anti-Rho blots) and incubated for $1 \mathrm{hr}$ at room temperature with the appropriate secondary antibody, which was horseradish peroxidase-conjugated sheep anti-mouse $\operatorname{Ig} G$ at a dilution of either 1:5000 (anti-FAK immunoblot) or $1: 2500$ (anti-paxillin immunoblot). A donkey anti-rabbit IgG conjugated to horseradish peroxidase at a dilution of $1: 2000$ was employed as secondary antibody in Rho-B immunoblots. Membranes were washed with several changes of T-TBS and processed for detection by enhanced chemiluminescence (ECL) according to the manufacturer's instructions. In all blots, bands corresponding to the protein of interest (FAK, paxillin, or Rho-B) were identified by reference to protein standards (markers) run in parallel and were scanned using a UMAX supervista S-12 scanner.

\section{Results}

Tyrosine Phosphorylation Is Involved in DEXInduced Actin Polymerization

To explore whether activation of a tyrosine kinase occurs in actin polymerization induced by DEX, Ishikawa cells were pretreated with two different tyrosine kinase inhibitors, genistein at $100 \mu \mathrm{g} / \mathrm{ml}$ for $90 \mathrm{~min}$ and erbstatin analogue (EA) at $1 \mu \mathrm{M}$ for $3 \mathrm{hr}$, and with an inhibitor of cellular phosphatases, pervanadate (1 $\mathrm{mM}$ sodium orthovanadate in conjunction with $3 \mathrm{mM}$ hydrogen peroxide), for $30 \mathrm{~min}$. Changes in the G-/total actin ratio were measured, following a 15-min treatment with $\operatorname{DEX}\left(10^{-7} \mathrm{M}\right)$. In all experiments, the total actin content did not change significantly. Figure 1 summarizes the obtained results. DEX significantly reduced the $\mathrm{G}$-/total actin ratio, indicating actin assembly. In the presence of genistein (Fig. 1A) or EA 


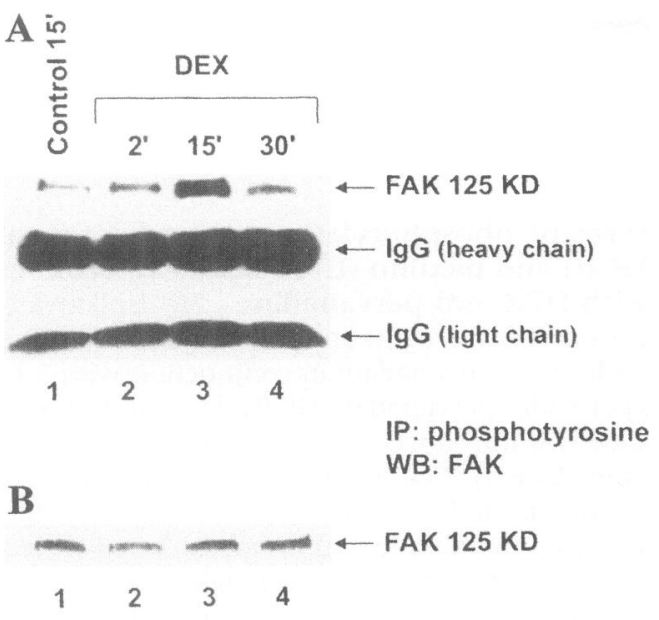

WB: FAK

Fig. 2. Tyrosine phosphorylation (A) and protein levels (B) of FAK in Ishikawa cells treated with DEX. (A) Ishikawa cells were serum starved overnight and then exposed to $10^{-7} \mathrm{M}$ DEX for 2 , 15 , and $30 \mathrm{~min}$. Cell lysates were immunoprecipitated with anti-phosphotyrosine monoclonal antibody and after separation of immunoprecipitates on SDS-polyacrylamide gradient gel $(4-20 \%)$, proteins were transferred to a nitrocellulose membrane and immunoblotted with anti-FAK monoclonal antibody. Heavy and light chains of the anti-phosphotyrosine IgG used to immunoprecipitate proteins phosphorylated on tyrosine residues are also shown. IP, immunoprecipitation, WB; Western blot. (B) FAK was immunoprecipitated from Ishikawa cells that were serum starved overnight and then incubated with DEX for 2, 15, and $30 \mathrm{~min}$. After separation of immunoprecipitates on SDS-polyacrylamide gradient gel $(4-20 \%)$, proteins were transferred to a nitrocellulose membrane and immunoblotted with anti-FAK monoclonal antibody.

(Fig. 1B), the DEX effect on actin polymerization was completely inhibited, since the G-/total actin ratios returned to control levels. Values were $0.46 \pm 0.0085$ (control, $n=6$ ); $0.345 \pm 0.01$ (DEX, $n=6) ; 0.47 \pm 0.026$ (genistein, $n=5$ ); $0.445 \pm 0.011$ (genistein + DEX, $n=5$ ); $0.448 \pm$ $0.023(\mathrm{EA}, n=6)$; and $0.46 \pm 0.022$ (EA + DEX, $n=5$ ). In the presence of pervanadate (Fig. 1C) the G-/total actin ratio was decreased significantly compared to control cells (values were $0.316 \pm 0.013, n=6$ vs. $0.46 \pm 0.0085, n=6)$. This decrease was more pronounced $(0.16 \pm$ $0.018, n=4$ ) following a 15 -min treatment with $\operatorname{DEX}\left(10^{-7} \mathrm{M}\right)$.

The ability of the tyrosine kinase inhibitors to prevent actin polymerization induced by DEX indicates that tyrosine kinases mediate this effect

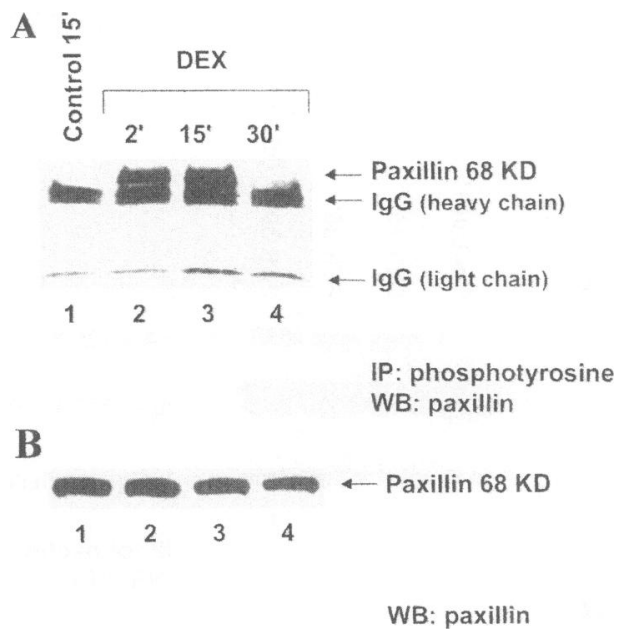

Fig. 3. Tyrosine phosphorylation (A) and protein levels (B) of paxillin in Ishikawa cells treated with DEX. (A) Ishikawa cells were serum starved overnight and then exposed to $10^{-7} \mathrm{M}$ DEX for 2, 15, and $30 \mathrm{~min}$. Cell lysates were immunoprecipitated with anti-phosphotyrosine monoclonal antibody, and after separation of immunoprecipitates on SDS-polyacrylamide gradient gel $(4-20 \%)$, proteins were transferred to a nitrocellulose membrane and immunoblotted with anti-paxillin monoclonal antibody. Heavy and light chains of the anti-phosphotyrosine IgG used to immunoprecipitate proteins phosphorylated on tyrosine residues are also shown. IP, immunoprecipitation, WB, Western blot. (B) Ishikawa cells were serum starved overnight and then treated with DEX for 2, 15, and $30 \mathrm{~min}$. After separation on SDS-polyacrylamide gel $(12 \%)$, proteins were transferred to a nitrocellulose membrane and immunoblotted with anti-paxillin monoclonal antibody.

of DEX. Moreover, the ability of the tyrosine phosphatase inhibitor to induce actin polymerization and to further enhance the DEX effect on actin polymerization indicates that the observed changes in the actin cytoskeleton of Ishikawa cells are linked to levels of tyrosine phosphorylation of specific proteins.

\section{DEX Induces Rapid and Transient FAK and Paxillin Tyrosine Phosphorylation}

We explored the effect of DEX on levels of expression and extent of tyrosine phosphorylation of FAK and paxillin, two cytoskeleton-associated proteins that promote assembly of actin microfilaments. To determine whether these cellular proteins are also substrates for DEX-induced actin polymerization, Ishikawa cells were incubated with DEX $\left(10^{-7} \mathrm{M}\right)$ for 2,15 , and $30 \mathrm{~min}$ and lysates of the treated cells were immuno- 


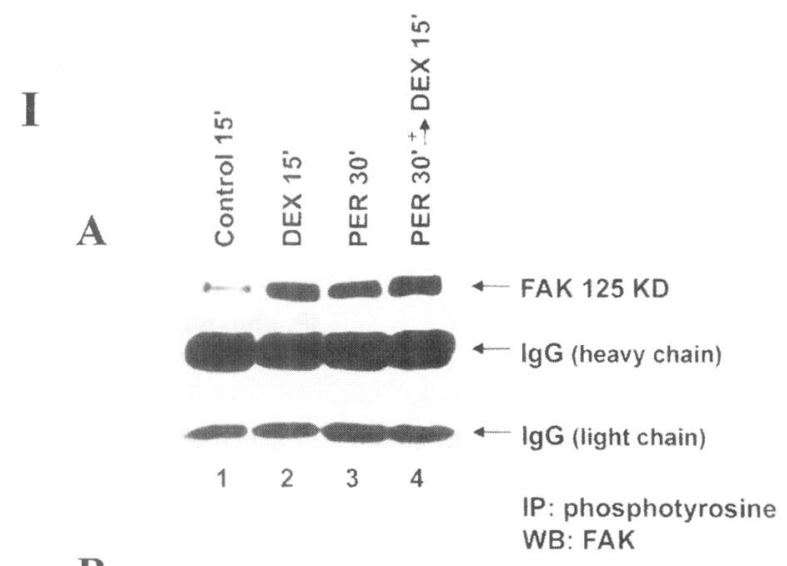

B

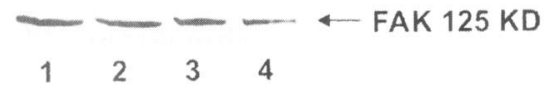

WB: FAK

II

A

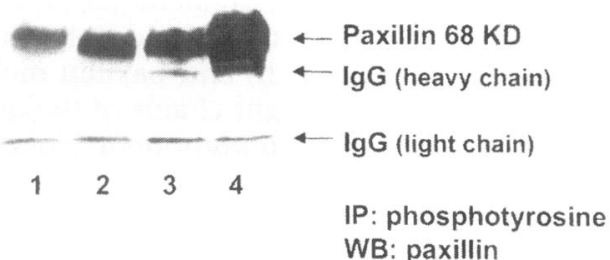

B

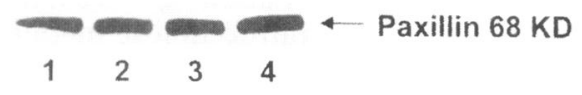

WB: paxillin
Fig. 4. Tyrosine phosphorylation (A) and expression (B) of FAK (I) and paxillin (II) in Ishikawa cells treated with DEX and pervanadate. (IA) Ishikawa cells were serum starved overnight and then exposed for $30 \mathrm{~min}$ to $1 \mathrm{mM}$ sodium orthovanadate in conjunction with $3 \mathrm{mM}$ hydrogen peroxide [pervanadate (PER), lane 3]. Subsequently, DEX was introduced at a final concentration of $10^{-7}$ $M$ for 15 min (lane 4). Cell lysates were prepared as described in Materials and Methods. Proteins were transferred to a nitrocellulose membrane and immunoblotted with antiFAK monoclonal antibody. Heavy and light chains of the anti-phosphotyrosine IgG used to immunoprecipitate proteins phosphorylated on tyrosine residues are also shown. IP, immunoprecipitation; WB, Western blot (IB) Experiment was performed as described in IA. Cell lysates were immunoprecipitated with anti-FAK monoclonal antibody, and after separation of immunoprecipitates on SDS-polyacrylamide gradient $(4-20 \%)$ gel, proteins were transferred to a nitrocellulose membrane and immunoblotted with anti-FAK monoclonal antibody. (IIA) Ishikawa cells were serum starved overnight and then exposed for $30 \mathrm{~min}$ to $1 \mathrm{mM}$ sodium orthovanadate in conjunction with $3 \mathrm{mM}$ hydrogen peroxide [pervanadate (PER), lane 3]. Subsequently, DEX was introduced at a final concentration of $10^{-7} \mathrm{M}$ for $15 \mathrm{~min}$ (lane 4). Cell lysates were prepared as described in Materials and Methods. Proteins were transferred to a nitrocellulose membrane and immunoblotted with anti-paxillin monoclonal antibody. Heavy and light chains of the anti-phosphotyrosine IgG used to immunoprecipitate proteins phosphorylated on tyrosine residues are also shown. IP, immunoprecipitation; WB, Western blot. (IIB) Experiment was performed as described in IIA Cell lysates were run on SDS-polyacrylamide gel (12\%), and proteins were transferred to a nitrocellulose membrane and immunoblotted with anti-paxillin monoclonal antibody.

precipitated with either anti-phosphotyrosine monoclonal antibody or the monoclonal antibody against FAK and paxillin, respectively. The immunoprecipitates were further analyzed by Western blotting.

Figure 2A shows that immunoprecipitated FAK was heavily phosphorylated on tyrosine after a 15-min incubation period. This increase in tyrosine phosphorylation of FAK was transient (not detectable at $30 \mathrm{~min}$ ). DEX had no effect on FAK protein levels (Fig. 2B). Figure 3A shows the changes in tyrosine phosphorylation of paxillin following a 2-, 15-, and 30-min incubation of Ishikawa cells with DEX $\left(10^{-7} \mathrm{M}\right)$. An early transient increase in tyrosine phosphorylation of paxillin was detectable after a 2- and 15-min incubation period. DEX had no effect on paxillin protein levels (Fig. 3B).

These findings indicate that glucocorticoids

induce rapid but transient tyrosine phosphorylation of FAK and paxillin, and implicate tyrosine phosphorylation of these proteins, an underlying mechanism that regulates the rapid alterations of actin microfilament dynamics following DEX treatment of human endometrial cells.

Inhibition of Cytosolic Tyrosine Phosphatases Mimics Effect of DEX on Tyrosine Phosphorylation of FAK and Paxillin and Enhances DEX Effect

To examine the effects of tyrosine phosphatase inhibition on DEX-induced tyrosine phosphorylation of FAK and paxillin, Ishikawa cells were incubated with $1 \mathrm{mM}$ sodium orthovanadate in conjunction with $3 \mathrm{mM}$ hydrogen peroxide for $30 \mathrm{~min}$, followed by a 15-min treatment with $\operatorname{DEX}\left(10^{-7} \mathrm{M}\right)$. Lysates of the treated cells were immunoprecipitated with either anti-phosphoty- 


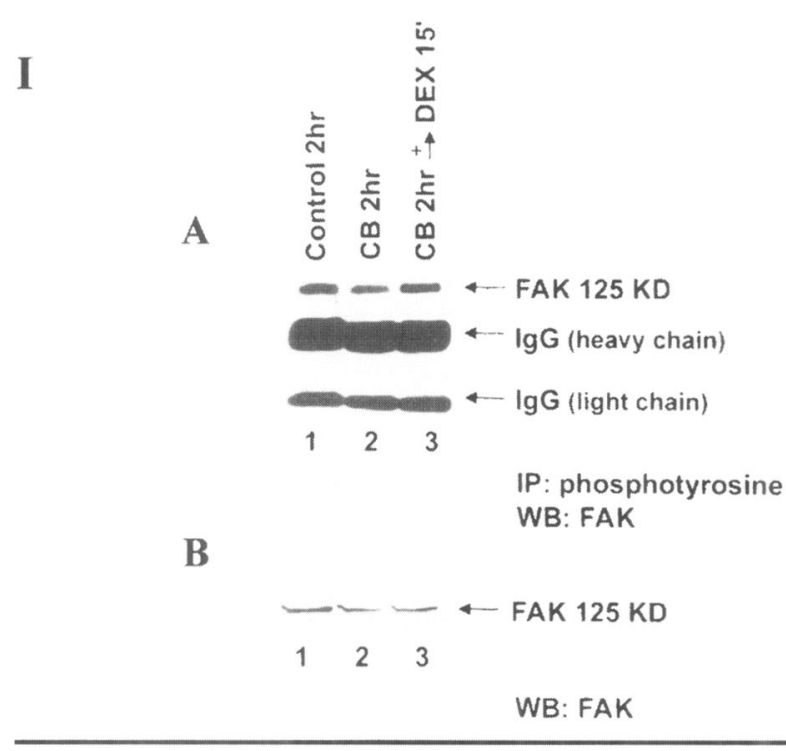

II

A

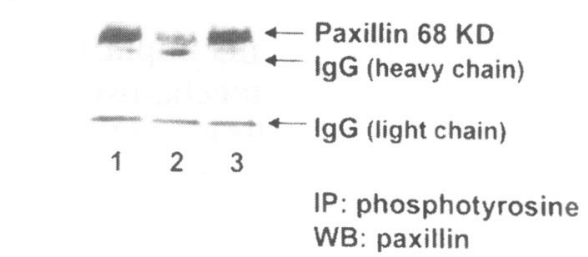

B WB: paxillin

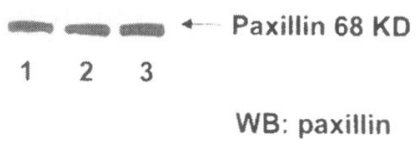

rosine monoclonal antibody or monoclonal antibody against FAK and paxillin, respectively. Immunoprecipitates were analyzed by Western blotting.

In pervanadate-treated cells there was an increase in FAK tyrosine phosphorylation, as shown in Figure 4IA (lane 3), to a level similar to that of DEX-treated cells (Fig. 4IA, lane 2). In cells treated with pervanadate for $30 \mathrm{~min}$ followed by a 15 -min treatment with DEX $\left(10^{-7} \mathrm{M}\right)$, the levels of tyrosine phosphorylation of FAK were markedly elevated (Fig. 4IA, lane 4). Figure 4IIA shows the effect of pervanadate on tyrosine phosphorylation of paxillin. Pervanadate increased tyrosine phosphorylation levels of paxillin (lane 3 ) to a level similar to that of DEX-treated cells (lane 2). In cells treated with pervanadate for $30 \mathrm{~min}$, followed by a $15-\mathrm{min}$ treatment with $\operatorname{DEX}\left(10^{-7}\right.$ $\mathrm{M})$, tyrosine phosphorylation levels of paxillin were markedly elevated. Pervanadate alone, DEX alone, or the combination of the two had no effect on FAK and paxillin protein levels
Fig. 5. Tyrosine phosphorylation (A) and expression (B) of FAK (I) and paxillin (II) following treatment of Ishikawa cells with cytochalasin B (CB) and DEX. (IA) Ishikawa cells were serum starved overnight and then treated with CB (1.2 $\mu \mathrm{M})$ for $2 \mathrm{hr}$. Subsequently, DEX was introduced at a final concentration of $10^{-7} \mathrm{M}$ for 15 min. Cell lysates were prepared as described in Materials and Methods. Proteins were transferred to a nitrocellulose membrane and immunoblotted with anti-FAK monoclonal antibody. Heavy and light chains of the anti-phosphotyrosine IgG used to immunoprecipitate proteins phosphorylated on tyrosine residues are also shown. IP, immunoprecipitation; WB, Western blot. (IB) Experiment was performed as described in IA. Cell lysates were immunoprecipitated with anti-FAK monoclonal antibody and after separation of immunoprecipitates on SDS-polyacrylamide gradient $(4-20 \%)$ gel, and proteins were transferred to a nitrocellulose membrane and immunoblotted with antiFAK monoclonal antibody. (IIA) Ishikawa cells were serum starved overnight and then treated with $\mathrm{CB}(1.2 \mu \mathrm{M})$ for $2 \mathrm{hr}$. Subsequently, DEX was introduced at a final concentration of $10^{-7} \mathrm{M}$ for $15 \mathrm{~min}$. Cell lysates were prepared as described in Materials and Methods. Proteins were transferred to a nitrocellulose membrane and immunoblotted with anti-paxillin monoclonal antibody. Heavy and light chains of the anti-phosphotyrosine IgG used to immunoprecipitate proteins phosphorylated on tyrosine residues are also shown. IP, immunoprecipitation; WB, Western blot. (IIB) Experiment was performed as described in IA. Cell lysates were run on SDS-polyacrylamide gel $(12 \%)$, and proteins were transferred to a nitrocellulose membrane and immunoblotted with anti-paxillin monoclonal antibody.

(Fig. 4IB and 4IIB). These data support the idea that the observed changes in the cytoskeleton of Ishikawa cells in the presence of glucocorticoids are related to the levels of tyrosine phosphorylation of two cytoskeleton-associated proteins-FAK and paxillin. Moreover, increasing tyrosine phosphorylation with some tyrosine phosphatase inhibitors not only leads to actin polymerization but also enhances glucocorticoid action.

DEX Restores Tyrosine Phosphorylation of FAK and Paxillin following Disruption of Cytoskeletal Assembly by $C B$

Actin stress fibers emanate from focal adhesions and link focal adhesions with the cytoskeleton. Since FAK and paxillin are both localized in focal adhesions, we examined whether the integrity of the actin filament network is necessary for DEXstimulated tyrosine phosphorylation of these proteins.

Ishikawa cells were pretreated for $2 \mathrm{hr}$ 
with $1.2 \mu \mathrm{M}$ of the cytoskeletal assembly disrupter $\mathrm{CB}$ and then stimulated with $10^{-7} \mathrm{M}$ DEX for $15 \mathrm{~min}$. Cell lysates were immunoprecipitated with anti-phosphotyrosine monoclonal antibody and analyzed by Western blotting using monoclonal antibody against FAK or against paxillin. Figure 5IA shows the effect of DEX on FAK tyrosine phosphorylation in the presence and absence of $C B$. In cells incubated with $1.2 \mu \mathrm{M} \mathrm{CB}$ for $2 \mathrm{hr}$ (lane 2), there was a decrease in FAK tyrosine phosphorylation levels compared to untreated (control) cells (lane 1). Addition of $10^{-7} \mathrm{M} \mathrm{DEX}$ for $15 \mathrm{~min}$ into cells that had been pretreated with $1.2 \mu \mathrm{M} C B$ for $2 \mathrm{hr}$ (lane 3 ) restored FAK tyrosine phosphorylation to control levels. The effect of DEX on paxillin tyrosine phosphorylation in the presence and absence of $\mathrm{CB}$ is shown in Figure 5IIA. In cells incubated with $1.2 \mu \mathrm{M} \mathrm{CB}$ for $2 \mathrm{hr}$ (lane 2) there was a decrease in paxillin tyrosine phosphorylation levels compared to untreated cells (lane 1). Addition of $10^{-7} \mathrm{M}$ DEX for 15 min into cells that had been pretreated with $1.2 \mu \mathrm{M} \mathrm{CB}$ for $2 \mathrm{hr}$ (lane 3) restored paxillin tyrosine phosphorylation to control levels. The 2 -hr incubation of cells with CB alone had no effect on FAK or paxillin protein levels, (Fig. 5IB and 5IIB). These data indicate that the integrity of the actin cytoskeleton is essential for tyrosine phosphorylation of FAK and paxillin. Moreover, they strongly support a role for glucocorticoids in stabilizing actin cytoskeleton (2), since DEX restored tyrosine phosphorylation of these proteins following disruption of the cytoskeletal assembly by $\mathrm{CB}$.

DEX Increases RhoB Protein Levels Only after LongTerm Treatment

The Ras-related GTP binding protein RhoB regulates actin cytoskeleton organization as well as expression and activity of FAK and paxillin $(21,24,25)$. Therefore, we assessed whether the observed increase in actin polymerization (Fig. 1) and tyrosine phosphorylation levels of FAK and paxillin (Figs. 2, 3A) was associated with an increase in RhoB levels in Ishikawa cells treated with DEX $\left(10^{-7} \mathrm{M}\right)$ for different time periods. Figure 6 shows a Western blot analysis of RhoB in cells treated with DEX for $3,6,12$, and $24 \mathrm{hr}$. No changes in RhoB expression were observed after 3 and $6 \mathrm{hr}$ of treatment with DEX compared to untreated (control) cells, whereas there was an important

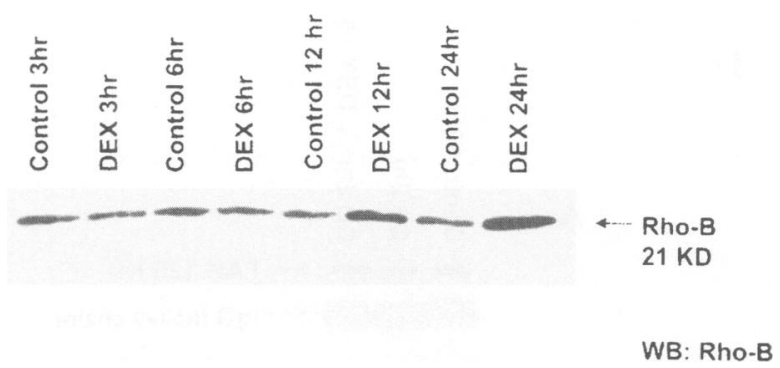

Fig. 6. Expression of Rho-B protein in DEXtreated cells. Ishikawa cells were serum starved overnight and then incubated with $10^{-7} \mathrm{M}$ DEX for $3,6,12$, and $24 \mathrm{hr}$. Cell lysates were separated on SDS-polyacrylamide gel (12\%), and proteins were transferred to a nitrocellulose membrane and immunoblotted with anti-RhoB polyclonal antibody. WB, Western blot.

increase in RhoB levels after 12 and $24 \mathrm{hr}$ of treatment. Our results implicate a Rho expression-independent mechanism for the shortterm increase in FAK and paxillin tyrosine phosphorylation by DEX. The long-term increase in Rho levels after steroid treatment may constitute part of the mechanism underlying the long-term stabilization effects of glucocorticoids on actin cytoskeleton (31).

\section{Discussion}

In previous studies $(2,3)$, we have shown that one of the mechanisms by which glucocorticoids induce rapid, nongenomic cellular events involves changes in the dynamics of actin polymerization. Using different experimental approaches, including microscopic analysis and quantitative determinations of the G-/total actin ratio and of F-actin levels, we analyzed the time course, the dose dependence, and the specificity of DEX action on the actin cytoskeleton of Ishikawa human endometrial cells. We showed that DEX induced actin assembly and microfilament stabilization within $15 \mathrm{~min}$, even in the presence of transcriptional inhibitor actinomycin $\mathrm{D}$. These findings were confirmed by confocal laser microscopy. Moreover, actin biosynthesis and the concentration of the actin transcript were unaffected, as showed by quantitative immunoblot and Northern blot analysis, respectively, which suggests that glucocorticoids induce a fast and self-limited polymerization of actin in human endometrial cells without affecting its synthesis. The molecular mechanisms of DEX-actin inter- 
action are not clear. However, the alterations in actin cytoskeleton dynamics by DEX were shown to be closely associated with rapid changes in cellular cAMP levels (2), which suggests involvement of regulatory molecules with kinase activity.

In this study, we explored putative mechanisms mediating the rapid, nongenomic effects of glucocorticoids. We first examined the role of tyrosine phosphorylation on DEX-induced actin assembly, using two dissimilar tyrosine kinase inhibitors-genistein and EA $(27,28)$-and determined changes in G-/total actin ratio in the presence and absence of DEX. Our observations indicate that both genistein and EA inhibited DEX-induced actin polymerization (Fig. 1A,B), providing indirect evidence that tyrosine phosphorylation is required for the DEX effect on actin assembly. Experimental findings using the protein tyrosine phosphatase inhibitor pervanadate support this suggestion. Sodium orthovanadate in conjunction with hydrogen peroxide (pervanadate) acts as a potent inhibitor of a variety of cellular phosphatases in vivo (7), and it causes rapid formation of focal adhesions in MDCK cells (32). In addition, increased tyrosine phosphatase activity has been associated with cell detachment from the substratum and focal adhesion disassembly (33). Indeed, pervanadate not only mimicked the effect of DEX on actin polymerization by decreasing the G-/total actin ratio but also enhanced DEX action when added prior to DEX (Fig. 1C). Taken together, the effects of tyrosine kinase and tyrosine phosphatase inhibition strongly suggest that signaling pathways involving tyrosine phosphorylation events are essential in mediating actin assembly by DEX.

These findings are in line with previous studies reporting that tyrosine phosphorylation is essential for microfilament assembly $(6,7)$. Proteins that become phosphorylated on tyrosine in association with microfilament assembly and formation of focal adhesions include FAK and paxillin (11). Increases in FAK and paxillin tyrosine phosphorylation are accompanied by profound alteration in the organization of actin cytoskeleton and in the assembly of focal adhesions, the distinct areas of plasma membrane where FAK and paxillin are localized (34). In addition, dephosphorylation of FAK and paxillin, i.e., by ethacrynic acid, disrupts signaling pathways that normally maintain the stability of the actin cytoskeleton and cellular adhesions (35). FAK and paxillin are frequently found to colocalize at the termini of F-actin fibers in focal adhesions $(36,37)$. FAK consists of a highly conserved ty- rosine kinase domain flanked by amino- and carboxyl-terminal noncatalytic domains. The carboxyl-terminal domain mediates localization of FAK to focal adhesions and binding of FAK to paxillin (38). Tyrosine phosphorylation of the cytosolic protein kinase FAK and of the cytoskeleton-associated protein paxillin has been identified as an early event in the action of diverse signaling molecules, including mitogenic neuropeptides (18), growth factors such as plateletderived growth factor (PDGF) $(16,17)$, the bioactive lipid LPA $(8,15)$, vasopressin, bombesin, and endothelin $(14,15)$, ECM proteins, $(11)$ and tumor necrosis factor $\alpha$ (TNF- $\alpha$ ) (39). FAK, in turn, phosphorylates paxillin $(19,20)$.

Our observations identify DEX as a stimulus for phosphorylation of both FAK and paxillin on tyrosine residues in Ishikawa cells. This was studied by immunoprecipitation-immunoblotting methods. Short-term incubation of cells with DEX transiently increased tyrosine phosphorylation of FAK and paxillin without affecting total protein levels. These findings indicate that glucocorticoids induce rapid tyrosine phosphorylation of these cytoskeleton-associated proteins and may represent a regulatory mechanism controlling the rapid alterations of actin microfilament dynamics following DEX treatment of human endometrial cells. This hypothesis is further supported by the finding that tyrosine phosphorylation is a transient event, a result in accordance with the fact that DEX-induced rapid actin polymerization is a transient phenomenon (2). Whether tyrosine phosphorylation of these proteins is required as a unique mechanism regulating the observed actin polymerization in response to DEX remains to be determined. However, the results provided by the tyrosine kinase inhibitors (Fig. 1A,B), although not strictly tyrosine kinase-specific, strongly suggest a central role for tyrosine phosphorylation in mediating rapid actin assembly and microfilament stabilization by DEX.

An alternative proposal to our observations is that DEX-induced actin polymerization is the primary event that mediates tyrosine phosphorylation of FAK and paxillin. In support of this contention is our finding that inhibition of actin polymerization by $\mathrm{CB}$, which selectively disrupts the actin filament network, abrogated tyrosine phosphorylation of FAK and paxillin by DEX, whereas DEX treatment restored tyrosine phosphorylation of both proteins following exposure to CB (Fig. 5IA, 5IIA). This is in agreement with similar observations in other systems in which cytochalasins inhibit both phosphorylation of 
FAK and paxillin and formation of focal adhesions $(13,15,39,40)$. In this regard, the observed actin polymerization induced by DEX may constitute a mechanism for transduction of signals that regulate tyrosine phosphorylation of FAK and paxillin and may indicate that an intact actin cytoskeleton is required for the transmission of signals. This idea is supported by a number of observations on signaling events under conditions of disruption of the actin cytoskeleton by cytochalasins. For example, transforming growth factor (TGF)- $\beta-1$, which regulates actin cytoskeleton organization, protects fibroblast cytoarchitecture from the actin destabilizing cytochalasins $\mathrm{B}$ and D (23). FAK or paxillin phosphorylation $(11,39,41)$ and MAP kinase activation after engagement of integrins in fibroblasts $(41,42)$ are blocked in the presence of $\mathrm{CB}$.

Evidence shows that there are bidirectional connections between signaling pathways and cytoskeleton. For example, the Ras-related, small GTP-binding proteins Rho and rac are involved in controlling actin polymerization, regulating activity of FAK and paxillin, and stimulating formation of focal adhesions between cells and their substratum (21-25). Moreover, Rho-induced assembly of focal adhesions and actin stress fibers can be blocked by the kinase inhibitor genistein, suggesting that an essential, rho-regulated tyrosine kinase is required (21). Also neuropeptide-mediated induction of tyrosine phosphorylation of FAK and paxillin is critically dependent on not only the integrity of the actin cytoskeleton but also functional Rho (18). A likely explanation for the apparent synchrony of focal adhesion and stress fiber formation is that actin polymerization and focal adhesion assembly are two distinct downstream effects of the same regulatory molecule, Rho $(24,25,43,44)$. Therefore, a possible explanation for the inhibitory effect of CB on both actin polymerization and FAK or paxillin phosphorylation induced by DEX may be that $\mathrm{CB}$ interferes with receptor-mediated activation of Rho (45). In an attempt to elucidate this, we studied RhoB expression in Ishikawa cells following treatment with DEX for different time periods. An increase in RhoB protein levels was noticed after a 12- and 24-hr treatment with DEX, whereas RhoB levels remained unchanged after 3 and $6 \mathrm{hr}$ of treatment (Fig. 6). Although these experiments can not exclude changes in the activity of this small GTPase, we can hypothesize that the increase in RhoB expression after long-term incubation with DEX may constitute a part of the mechanism for the reported long-term action of glucocorticoids on actin cytoskeleton (31). Con- versely, a Rho-independent mechanism is most probably implicated in the DEX-induced shortterm increase in actin polymerization and FAK and paxillin tyrosine phosphorylation.

In summary, our observations suggest that in human endometrial cells, tyrosine phosphorylation of the cytoskeleton-associated proteins FAK and paxillin accompanies the rapid effect of DEX on actin assembly. Tyrosine kinases may represent a more general regulatory mechanism of rapid actin polymerization and reorganization, induced by various extracellular signals. Indeed, it appears that, in addition to glucocorticoids, TNF- $\alpha$ may have similar effects on paxillin and FAK phosphorylation, associated with rapid actin reorganization (39). Our findings support the notion that rapid actin cytoskeleton redistribution, regulated by tyrosine phosphorylation signaling mechanisms, may be an important step in controlling the signal transduction pathway of the nongenomic glucocorticoid response. Moreover, the long-term increase in RhoB expression after DEX treatment is compatible with the recently proposed dual model for steroid action, which addresses both the rapid nongenomicand the genomic-mediated effects (1-3).

In conclusion, we suggest the existence of a signal transduction link that involves FAK and paxillin tyrosine phosphorylation, assembly, and stabilization of actin structures and formation of focal adhesions. This link could act as a sensor and as a mediator of nongenomic steroid hormone signals for the modulation of cellular responses. Elucidation of the precise cause-effect relationships within this putative signal transduction pathway warrants further work.

\section{Acknowledgments}

The authors would like to thank Dr. E. A. Papakonstanti for many helpful comments. This work was supported by a grant from the Hellenic Pasteur Institute to C.S.

\section{References}

1. Wehling M. (1994) Nongenomic actions of steroid hormones. Trends Endocrin. Met. 5: 347-353.

2. Koukouritaki SB, Theodoropoulos PA, Margioris AN, Gravanis A, Stournaras C. (1996) Dexamethasone alters rapidly actin polymerization dynamics in human endometrial cells: evidence for nongenomic action involving cAMP turnover. $J$ Cell Biochem 62: 251-261. 
3. Koukouritaki SB, Margioris AN, Gravanis A, Hartig R, Stournaras C. (1997) Dexamethasone induces rapid actin assembly in human endometrial cells without affecting its synthesis. $J$ Cell Biochem 65: 492-500.

4. Kiley SC, Parker PJ, Fabbro D, Jaken S. (1992) Hormone- and phorbol ester-activated protein kinase $C$ isozymes mediate a reorganization of the actin cytoskeleton associated with prolactin secretion on $\mathrm{GH}_{4} \mathrm{C}_{1}$ cells. Mol Endocrinol 6: 120-131.

5. Moustakas A, Theodoropoulos PA, Gravanis A, Haussinger D, Stournaras C. (1998) The cytoskeleton in cell volume regulation. Contrib Nephrol 123: $121-134$.

6. Melamed I, Downey GP, Roifman CM. (1991) Tyrosine phosphorylation is essential for microfilament assembly in B-lymphocytes. Biochem Biophys Res Commun 176: 1424-1429.

7. Pumiglia KM, Lau L-F, Huang C-K, Burroughs S, Feinstein MB. (1992) Activation of signal transduction in platelets by the tyrosine phosphatase inhibitor pervanadate (vanadyl hydroperoxide). Biochem J 286: 441-449.

8. Chrzanowska-Wodnicka M, Burridge K. (1994) Tyrosine phosphorylation is involved in reorganization of the actin cytoskeleton in response to serum or LPA stimulation. J Cell Sci 107: 3643-3654.

9. Burridge K, Fath K, Kelly T, Nuckolls G, Turner C. (1988) Focal adhesions: transmembrane junctions between the extracellular matrix and the cytoskeleton. Annu Rev Cell Biol 4: 487-525.

10. Jaken S, Leach K, Klauck T. (1989) Association of type 3 protein kinase $C$ with focal contacts in rat embryo fibroblasts. J Cell Biol 109: 697-704.

11. Burridge K, Turner CE, Romer LH. (1992) Tyrosine phosphorylation of paxillin and ppl $25^{\mathrm{FAK}}$ accompanies cell adhesion to extracellular matrix: a role in cytoskeletal assembly. J Cell Biol 119: 893-903.

12. Clark EA, Brugge JS. (1995) Integrins and signal transduction pathways: the road taken. Science 268: 223-239.

13. Rozengurt E. (1995) Convergent signalling in the action of integrins, neuropeptides, growth factors and oncogenes. Cancer Surv 24: 81-96.

14. Zachary I, Sinnett-Smith J, Turner CE, Rozengurt E. (1993) Bombesin, vasopressin and endothelin rapidly stimulate tyrosine phosphorylation of the focal adhesion-associated protein paxillin in Swiss 3T3 cells. J Biol Chem 268: 22060-22065.

15. Rodriguez-Fernandez JL, Rozengurt E. (1998) Bombesin, vasopressin, lysophosphatidic acid and sphingosylphosphorylcholine induce focal adhesion kinase activation in intact Swiss 3T3 cells. $J$ Biol Chem 273: 19321-19328.

16. Rankin S, Rozengurt E. (1994) Platelet-derived growth factor modulation of focal adhesion kinase (ppl25 $5^{\mathrm{FAK}}$ ) and paxillin tyrosine phosphorylation in Swiss 3T3 cells. $J$ Biol Chem 269: 704-710.

17. Kim B, Feldman EL. (1998) Differential regulation of focal adhesion kinase and mitogen-activated protein kinase tyrosine phosphorylation during insulin-like growth factor-I-mediated cytoskeletal reorganization. J Neurochem 71: 1333-1336.

18. Rozengurt E. (1998) Gastrointestinal peptide signaling through tyrosine phosphorylation of focal adhesions proteins. Am J Physiol 275: G177-G182.

19. Turner CE, Schaller MD, Parsons JT. (1993) Tyrosine phosphorylation of the focal adhesion kinase ppl $25^{\mathrm{FAK}}$ during development: relation to paxillin. $J$ Cell Sci 105: 637-645.

20. Bellis SL, Miller JT, Turner CE. (1995) Characterization of tyrosine phosphorylation of paxillin in vitro by focal adhesion kinase. $J$ Biol Chem 270: 17437-17441.

21. Ridley AJ, Hall A. (1994) Signal transduction pathways regulating rho-mediated stress fibre formation: requirement for a tyrosine kinase. $E M B O$ $J$ 13: $2600-2610$.

22. Ridley AJ, Hall A. (1992) The small GTP-binding protein rho regulates the assembly of focal adhesions and actin stress fibers in response to growth factors. Cell 70: 389-399.

23. Moustakas A, Stournaras C. (1999) Regulation of actin cytoskeleton by TGF- $\beta$ in H-ras transformed fibroblasts. J Cell Sci 112: 1169-1179.

24. Tapon N, Hall A. (1997) Rho, rac and cdc42 GTPases regulate the organization of the actin cytoskeleton. Curr Opin Cell Biol 9: 86-92.

25. Flinn HM, Ridley AJ. (1996) Rho stimulates tyrosine phosphorylation of focal adhesion kinase, p130 and paxillin. J Cell Sci 109: 1133-1141.

26. Gravanis A, Gurpide E. (1986) Effects of estradiol on DNA polymerase a activity in a human endometrial adenocarcinoma cell line (Ishikawa cells). $J$ Clin Endocrinol Metab 63: 356-359.

27. Akiyama T, Ishida J, Nakagawa S, et al. (1987) Genistein, a specific inhibitor of tyrosine-specific protein kinases. $J$ Biol Chem 262: 5592-5595.

28. Bishop WR, Petrin J, Wang L, Ramesh U, Doll RJ. (1990) Inhibition of protein kinase C by the tyrosine kinase inhibitor erbstatin. Biochem Pharmacol 40: 2129-2135.

29. Katsantonis J, Toska A, Koukouritaki SB, Theodoropoulos PA, Gravanis A, Stournaras C. (1994) Differences in the G/total actin ratio and microfilament stability between normal and malignant human keratinocytes. Cell Biochem Funct 12: 267-274.

30. Faulstich H, Merkler I, Blackholm H, Stournaras C. (1984) Nucleotide in monomeric actin regulates the reactivity of the thiol groups. Biochemistry 23: 1608-1612.

31. Castellino F, Heuser J, Marchetti S, Bruno B, Luini A. (1992) Glucocorticoid stabilization of actin filaments: a possible mechanism for inhibition of corticotropin release. Proc Natl Acad Sci USA 89: 3775-3779.

32. Volberg T, Zick Y, Dror R, et al. (1992) The effect of tyrosine-specific protein phosphorylation on 
the assembly of adherens-type junctions. EMBO J 11: 1733-1742.

33. Maher PA. (1993) Activation of phosphotyrosine phosphatase activity by reduction of cell-substrate adhesion. Proc Natl Acad Sci USA 90: $11177-11181$.

34. Seufferlein T, Rozengurt E. (1995) Sphingosylphosphorylcholine rapidly induces tyrosine phosphorylation of $\mathrm{pl} 25^{\mathrm{FAK}}$ and paxillin, rearrangement of the actin cytoskeleton and focal actin assembly. $J$ Biol Chem 270: 24343-24351.

35. O'Brien ET, Kinch M, Harding TW, Epstein DL. (1997) A mechanism for trabecular meshwork cell retraction: ethacrynic acid initiates the dephosphorylation of focal adhesion proteins. Exp Eye Res 65 4: 471-483.

36. Tachibana K, Sato T, D'Avirro N, Morimoto C. (1995) Direct association of pp125 $5^{\mathrm{FAK}}$ with paxillin, the focal adhesion-targeting mechanism of pp125 $5^{\mathrm{FAK}}$. $J$ Exp Med 182: 1089-1100.

37. Turner CE, Glenney JR, Burridge K. (1990) Paxillin: a new vinculin-binding protein present in focal adhesions. J Cell Biol 111: 1059-1068.

38. Schaller MD, Parsons JT. (1994) Focal adhesion kinase and associated proteins. Curr Opin Cell Biol 6: 705-710.

39. Koukouritaki SB, Vardaki EA, Papakonstanti EA,
Lianos E, Stournaras C, Emmanouel DS. (1999) TNF- $\alpha$ induces actin cytoskeleton reorganization in glomerular epithelial cells involving tyrosine phosphorylation of paxillin and focal adhesion kinase. Mol Med 5: 382-392.

40. Melamed I, Turner CE, Aktories K, Kaplan DR, Gelfand EW. (1995) Nerve growth factor triggers microfilament assembly and paxillin phosphorylation in human B lymphocytes. $J$ Exp Med 181: 1071-1079.

41. Rosales C, O'Brien V, Kornberg L, Juliano R. (1995) Signal transduction by cell adhesion receptors. Biochim Biophys Acta 1242: 77-98.

42. Chen $Q$, Kinch MS, Lin TH, Burridge $K$, Juliano RL. (1994) Integrin-mediated cell adhesion activates mitogen-activated protein kinases. $J$ Biol Chem 269: 26602-26605.

43. Hanks SK, Polte TR. (1997) Signaling through focal adhesion kinase. Bioessays 19: 137-145.

44. Symons M. (1996) Rho family GTPases: the cytoskeleton and beyond. Trends Biochem Sci 21: 178-181.

45. Nobes CD, Hall A. (1995) Rho, rac and cdc42 GTPases regulate the assembly of multimolecular focal complexes associated with actin stress fibers, lamellipodia and filopodia. Cell 81: 53-62. 